\title{
A MULTI-OBJECTIVE OPTIMISATION MODEL FOR SEWER REHABILITATION CONSIDERING CRITICAL RISK OF FAILURE
}

\author{
Ben Ward*** and Dragan A. Savić** \\ * AECOM, Belverdere House, Pynes Hill, Exeter EX2 5WS (E-mail: ben.ward@aecom.com) \\ ** Centre for Water Systems, University of Exeter, North Park Road, Exeter EX4 4QF, UK. (E-mail: D.Savić@ex.ac.uk)
}

\begin{abstract}
A unique methodology for the optimal specification of sewer rehabilitation investment is presented in this paper. By accounting for the critical risk of asset failure, this methodology builds on previously successful work which explored the application of multi-objective optimisation tools to assist engineers with the specification of optimal rehabilitation strategies. The conventional sewerage rehabilitation specification process relies on the expertise of professional engineers to manually evaluate CCTV inspection information when determining the nature and extent of the rehabilitation solution. This process is not only tedious and subjective but it has no quantifiable means of identifying optimal solutions, or, possible combinations of optimal solutions in the delivery of catchment wide rehabilitation programmes. Therefore, the purely manual process of sewer rehabilitation design leaves a number of unanswered questions, such as: (1) Does the solution offer the greatest structural benefit to the network? (2) Is the solution the most cost effective solutions available? And, (3) Does the solution most greatly reduce the risk of critical asset failure? The application of a multi-objective genetic algorithm optimisation model, coupled with an enhanced critical risk methodology, has successfully answered these questions when applied to a case study data set provided by South West Water (UK).
\end{abstract}

Keywords: sewer rehabilitation, asset management, sewer, strategies, planning, critical risk, criticality, optimisation

\section{INTRODUCTION}

Most sewerage networks are compiled of ageing asset that are becoming increasingly more susceptible to failure. Abraham \& Gillani (1999) categories sewer system failure into three distinct modes; hydraulic, environmental and structural. Structural failure can have varying degrees of severity, ranging from minor sewer fabric defects, such as cracking, to complete loss of structural integrity where a full or partial collapse may be observed. The failure rate, or collapse rate, within a network is one of the major indicators that a sewerage system is deteriorating. In the most recent Asset Management Plan (AMP) submissions, nearly all of the UK's 10 water and sewerage utility companies have demonstrated a commitment to improve their sewerage asset failure rate (Ofwat, 2009). This is normally achieved through increased investment towards sewerage asset maintenance. To ensure that this increased level of investment returns the highest possible benefit, it will be crucial for comprehensive sewerage rehabilitation strategies to be developed and implemented over the next few years.

Only recently have authors begun to report on the application of Hydroinformatic tools to the problem of optimal sewerage asset management (Adey et al., 2003, and Elachachi \& Breysse, 2007). In comparison, methodologies addressing the optimal management of water distribution systems have been widely reported for numerous years, (Shamir \& Howard, 1979, Woodburn et al., 1987, Kim \& Mays, 1994, Halhal et al., 1997, and Malandain et al., 1998). If we purely consider the amount of published literature in the two fields, it would appear that the management of sewerage assets is less suited to the application of such Hydroinformatic tools. However, it will be shown here that the sewerage industry is in fact well suited to take advantage of such Hydroinformatic tools. In particular, the specification of optimal rehabilitation strategies can be improved through the increased use of structural condition grading information to inform decision making processes. In this respect, sewers have a distinct advantage over their clean water asset counterparts; in that their condition can be 
To be cited as: Ward, B. and D.A. Savić (2013) A multi-objective optimisation model for sewer rehabilitation considering critical risk of failure, Water Science \& Technology, Vol 66, No 11, pp 2410-2417.

ascertained relatively efficiently (Feeney, Thayer, et al., 2009 and Kathula et al., 1999), which can utilised for the implementation of prioritised maintenance and rehabilitation strategies (Newton \& Vanier, 2006).

\section{METHODS}

The multi-objective optimisation model presented in this paper utilises the standard sewer condition classification grading information obtained from CCTV inspections undertaken in-line with the current WRc (2004) Method of Sewer Condition Classification (MSCC4), to identify optimal rehabilitation solutions in terms of three conflicting objectives:

1. Maximise structural condition improvement;

2. Reduce construction cost; and

3. Minimise critical asset risk of failure.

A data management process and optimisation environment has been specifically developed to solve for these three conflicting objectives that are associated with the optimal specification of sewer rehabilitation solutions, shown in Figure 1. The optimisation environment is based upon a genetic algorithm (GA) approach, which is now a mature technology often used in water and wastewater planning and management (Nicklow et al., 2010). Genetic algorithms stem from the field of evolutionary computation and they are widely used within multi disciplinary industries to generate solutions to search and optimisation problems by mimicking behaviours found in biological evolution, namely, survival of the fittest, cross-over and mutation.

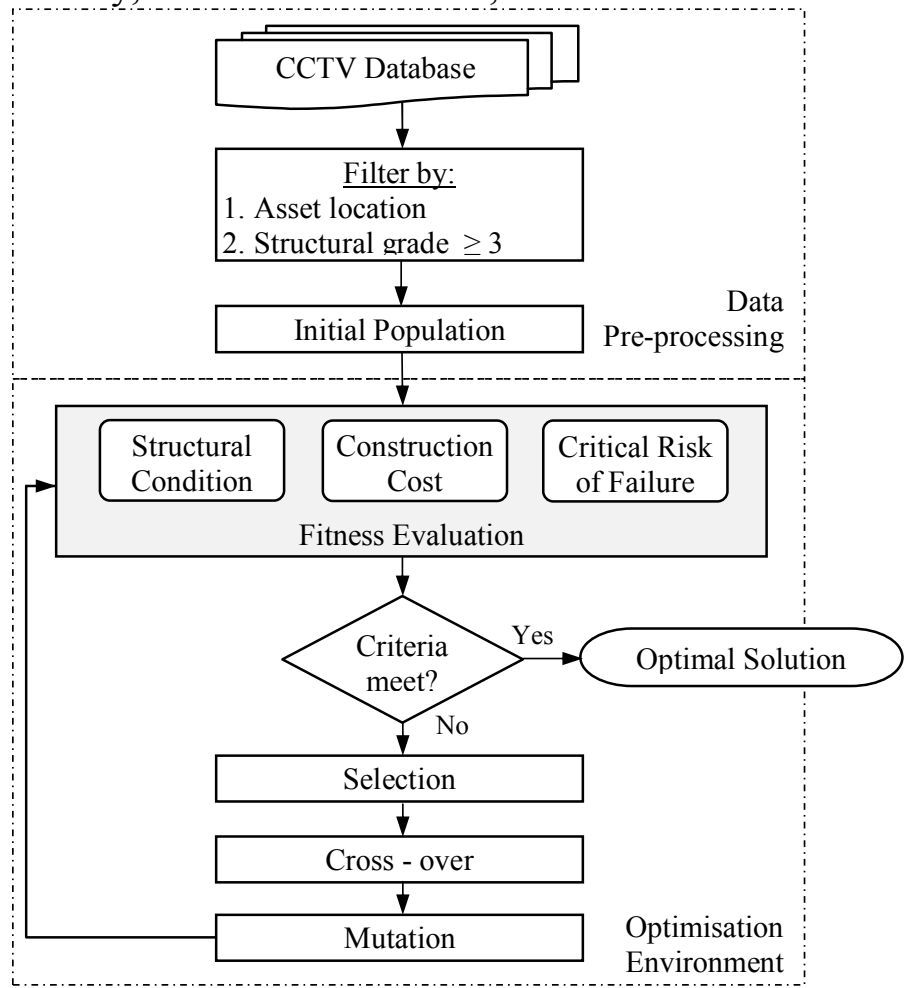

Figure 1: Data processing and optimisation environment

Data Pre-processing. The CCTV data is managed and coded using a commercial software package InfoNet developed by Innovyze (2010). InfoNet is used as a data pre-processing tool because of its geospatial data storage functionality and its ability to calculate structural and service condition grade information from raw CCTV data. Post data loading and condition coding, the CCTV database is 
To be cited as: Ward, B. and D.A. Savić (2013) A multi-objective optimisation model for sewer rehabilitation considering critical risk of failure, Water Science \& Technology, Vol 66, No 11, pp 2410-2417.

interrogated to remove structurally sound assets from being passed forward for consideration by the optimisation model. The optimisation model is driven by the extracted survey data which is directly processed within the optimisation environment to permit the application of a multi-objective optimisation algorithm.

Optimisation environment. The optimisation environment uses a macro driven programme to collate and present the raw CCTV into a format which allows the assignment of rehabilitation solutions to each segment of asset. The macro undertakes this process by creating a "chainage" (distance) value at increments along the assets. In this example, the term "chainage" is used to describe an imaginary line that is used to measure distance along a sewer. In sewer survey measurements, the chainage starts at $0 \mathrm{~m}$ in the starting manhole and observations are reported at $0.1 \mathrm{~m}$ increments until the end manhole is reached, or, the survey is terminated, e.g., a collapse or blockage is observed. Within the optimisation environment, the chainage is defined to $1.0 \mathrm{~m}$ accuracies which have been termed as segments. Whilst it is recognised that rehabilitation solutions can be delivered to accuracies of $0.1 \mathrm{~m}$, which are equal to the resolution obtained from the condition inspection equipment, it was deemed more practical to define the accuracy of the model in accordance with the minimum isolated repair length, i.e., $1 \mathrm{~m}$. Therefore, within each $1 \mathrm{~m}$ segment the accumulative structural score is calculated and displayed. The objective function formula, embedded in the optimisation spreadsheet template, then updates itself to encompass the cell ranges for each individual sewer length. Thus permitting the evaluation of the objective function(s) at asset level, i.e., the score improvement, construction costs and risk of failure can be considered per individual sewer length. In accordance with this level of accuracy, the condition of each $1 \mathrm{~m}$ segment of sewer is represented as the sum value of the defect scores within that meter. Similarly, for practical purposes, a minimum repair length of $1 \mathrm{~m}$ is adopted.

Optimisation tool. This model uses a well established GA optimisation tool developed by the University of Exeter, GANetXL, (Bicik et al., 2006; Savić et al., 2011). The optimisation model evaluates numerous rehabilitation solutions within the optimisation environment using a multiobjective GA. Upon establishing the optimisation environment, the GA assigns an initial random population of solutions, as a string of 1's and 0's, against each segment of the sewer. These 1's and 0's become the decision variables in the problem which represent the rehabilitation action; either rehabilitate (1) or do nothing (0). After an initial random population of decision variables are assigned, the GA evaluates the fitness of each solution based on the objective function(s) scores which are calculated dependent upon the decision variable values. If the fitness of the solution meets the stopping criteria for the algorithm then the optimal solution is said to be found. However, if the solution falls short of the criteria then the following GA operators are performed; selection, cross-over, mutation (Murphy et al., 1993) and the new solutions are re-evaluated. An important aspect in the optimisation process is how the algorithm uses the objective function(s) to prescribe the optimality of a particular solution, such that solutions can be ranked against one-another. The main advantage of this approach is the ability of a GA to find a set of Pareto-optimal (trade off) solutions in a single run of the algorithm.

\section{Model development}

Between 1980 and early 2000, UK water and sewerage companies largely employed asset management policies that involved the implementation of selective rehabilitation. Selective rehabilitation meant that non-critical sewers (Category C) would only receive maintenance on a reactive basis (Fenner et al., 2000). As we observe a behavioural shift in the industry towards more proactive maintenance, utility companies are becoming increasingly more and more concerned with the identification of critical assets, due to the high associated costs to the business when failure of said assets occur. Modern deterioration models that task themselves with the identification of defective assets are not typically 
founded on the use of asset criticality as a primary means for investigation (Berardi et al., 2008 and Black et al., 2005). Instead, other factors that might contribute to the observation of different deterioration rates amongst sewerage assets are often used, i.e., material, age and ground conditions (Davies, Clark, et al., 2001). Indeed, the use of criticality is often considered as an additional decision support element, used for evaluating the consequence element of the risk of failure. Whilst the identification of defective assets should not be prioritised towards critical sewers, it would be fundamentally flawed if a rehabilitation strategy were developed which considered the consequence of failure of critical and non-critical assets as equal. Thus, a balance must be struck to ensure that assets of equal likelihood of failure are rehabilitated in order of priority, i.e., where a bias towards the rehabilitation of critical sewers is given. This can be achieved using criticality as a surrogate measure to define the consequence of failure. It is important, however, that non-critical assets displaying characteristics of high failure probability are not neglected; hence the use of a bias function such as a criticality weighting is used instead of a blanket ruling for the repair of critical asset first.

\section{Objective functions}

Structural condition improvement objective function. The MSCC4 coding system developed by the WRc (2004), assigns a structural condition score to all structural defects that are observed along the asset's length during a condition inspection. The score value given to each observation is directly proportional to the severity of the defect observed. The optimisation tool utilizes this scoring system to evaluate the structural condition of the asset prior to and post different intervention scenario's.

Rehabilitation cost objective function. The cost objective is one of the most important elements in this model. It successfully allows the comparison of numerous different rehabilitation solutions against one another, in order to determine which solutions are more financially favourable than others. For example, the model can be tailored to distinguish between different contractor costs; where one contractor's rates may favour contiguous lining, as opposed to another, whose rate favours patch repairs. If these costs are accurately captured in the model the outputs will promote solutions according to each of the contractor's individual preferences. Similarly, the client may over-rule the objective function to promote their preference. To facilitate the accurate modelling of rehabilitation costs the total cost of any rehabilitation strategy is a function of the raw unit rate cost(s), client specific on$\operatorname{cost}(\mathrm{s})$ and the contractor mobilisation cost(s) associated with the repair or combination of repairs.

Critical risk of failure objective function. Risk is an important element to consider in any sewer rehabilitation strategy. Kaplan \& Garrick (1981) acknowledge that risk considers the likelihood of something occurring and the consequence of such an occurrence. This paper adopts a "critical risk" philosophy which attempts to model risk by understanding and evaluating the likelihood of a sewer failing and the consequence of that failure. The optimisation model uses the peak structural score observed for each asset, under the MSCC4 coding system, as a surrogate measure for the likelihood of failure. The methodology used to evaluate a sewers' criticality uses five consequence grades which are defined by a system that broadly follows the criticality grading guidance set-out in the Sewer Risk Manual (SRM), (WRc, 2004). Following this guidance, a sewerage asset is termed "critical" if the collapse or repair of the asset is either disruptive, expensive and/or if the asset is deemed to be of strategic importance. The UK Water Industry uses three criticality categories to distinguish between the different impacts of asset failure: Category " $A$ " refers to sewers where the cost of rehabilitation post asset failure would typically be in excess of double the planned renewal costs; Category "B" identifies assets where the cost of failure is less than " $\mathrm{A}$ ", but where the associated disruptions caused by collapse would make failure of these assets less desire able; And Category "C" applies to assets that 
are deemed non-critical from the above criteria. It is typical for Category "A" assets to possess any of the following characteristics; large diameter, greater than $3 \mathrm{~m}$ deep, beneath traffic sensitive streets, in bad ground, present troublesome access conditions and/or lie in close proximity to other infrastructure assets. The consequence based criticality approach used in this paper applies a further level of granularity to the existing approach by allowing for the original sewer criticality codes, "A" and " $\mathrm{B}$ ", to be escalated by the appendment of an " $\mathrm{x}$ ". The appendment is applied where the consequence of failure is deemed to be of significant importance, for example where asset failure would almost undoubtedly cause disruption to surrounding critical infrastructure, i.e., railways or major roads. The consequence criteria, which is applied via the use of Geographical Information System (GIS) analysis tools, is listed in Table 1.

Table 1: Example consequence criticality definitions

\begin{tabular}{lc}
\hline Code & Description of Sewer Category \\
\hline Ax & Under Railway \\
& $\begin{array}{c}\text { Under Motorway/Protected Street Sdf } \\
\text { Under Traffic Sensitive Street } \\
\text { Would Disrupt Hospital Traffic } \\
\text { Would Disrupt Fire Station Access }\end{array}$ \\
\hline A & WRc (2004) SRM sewer criticality classification \\
\hline Bx & Would Pollute Class 1 or 2 Main River \\
\hline B & In Hospital Complex \\
\hline C & Under Traffic Sensitive Street \\
\hline
\end{tabular}

\section{RESULTS}

A unique methodology for the optimal specification of sewer rehabilitation investment is presented in this paper. By accounting for the critical risk of asset failure during the specification of rehabilitation solutions, this methodology builds on the recent work of (Ugarelli \& Di Federico, 2010, and Ward \& Savić, 2010) which has reported notable benefits in the use of optimisation tools to assist engineers in the specification of optimal rehabilitation strategies. To ascertain the effectiveness of this approach a catchment case study is considered. The CCTV data used in the study originates from a recent sewer rehabilitation project implemented by South West Water, UK. The objective of the project was to use a deterioration model to efficiently target CCTV survey investigations within catchments leading to the identification and rehabilitation of defective sewers. The case study catchment has a total sewerage network of $37.4 \mathrm{~km}$, of which circa. $8 \%(2.9 \mathrm{~km})$ was surveyed using South West Water's targeted deterioration model. The effectiveness of the deterioration model successfully identifying sewers in a defective condition is evident in the structural condition grade summary, Figure 2, which shows a high percentage of structural condition grade 4 and 5 sewers from these targeted investigations. 


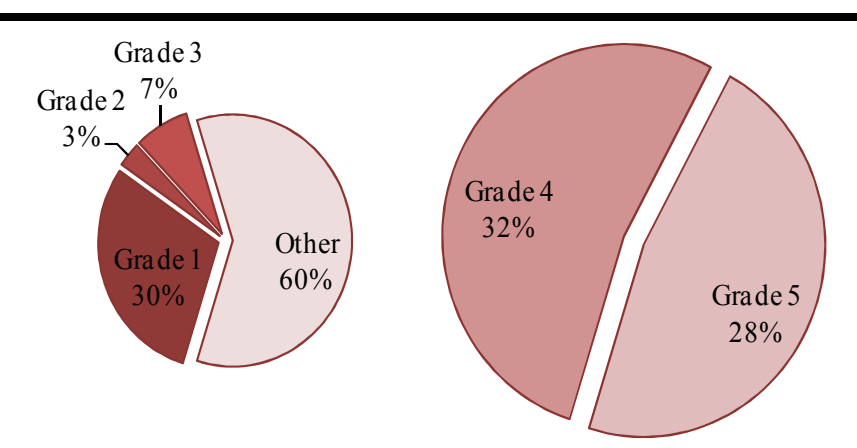

Figure 2: Structural condition profile summary

An initial filtering process was conducted outside of the optimisation environment to remove all structural sound assets from consideration by model. Approximately $60 \%(1.727 \mathrm{~km})$ of the raw CCTV data was passed forward into the optimisation modelling environment.. The key characteristics of the $1,727 \mathrm{~m}$ of data which includes; sewer age, diameter, material and criticality classification, is presented in Figure 3. Read \& Vickeridge (1997) estimate that $78 \%$ of all sewers in England and Wales are constructed from Vitrified Clay. Therefore, given the relatively small nature of the catchment, it is not surprising that the majority of the surveyed assets in this study are less than $225 \mathrm{~mm}$ diameter Vitrified Clay sewers. The high volume of sewers populating the oldest age band, i.e., pre 1896, is also typical of a data set founded on structurally defective condition surveys. Importantly, this data has sewers spanning all of the enhanced criticality classification codes from Ax - C.

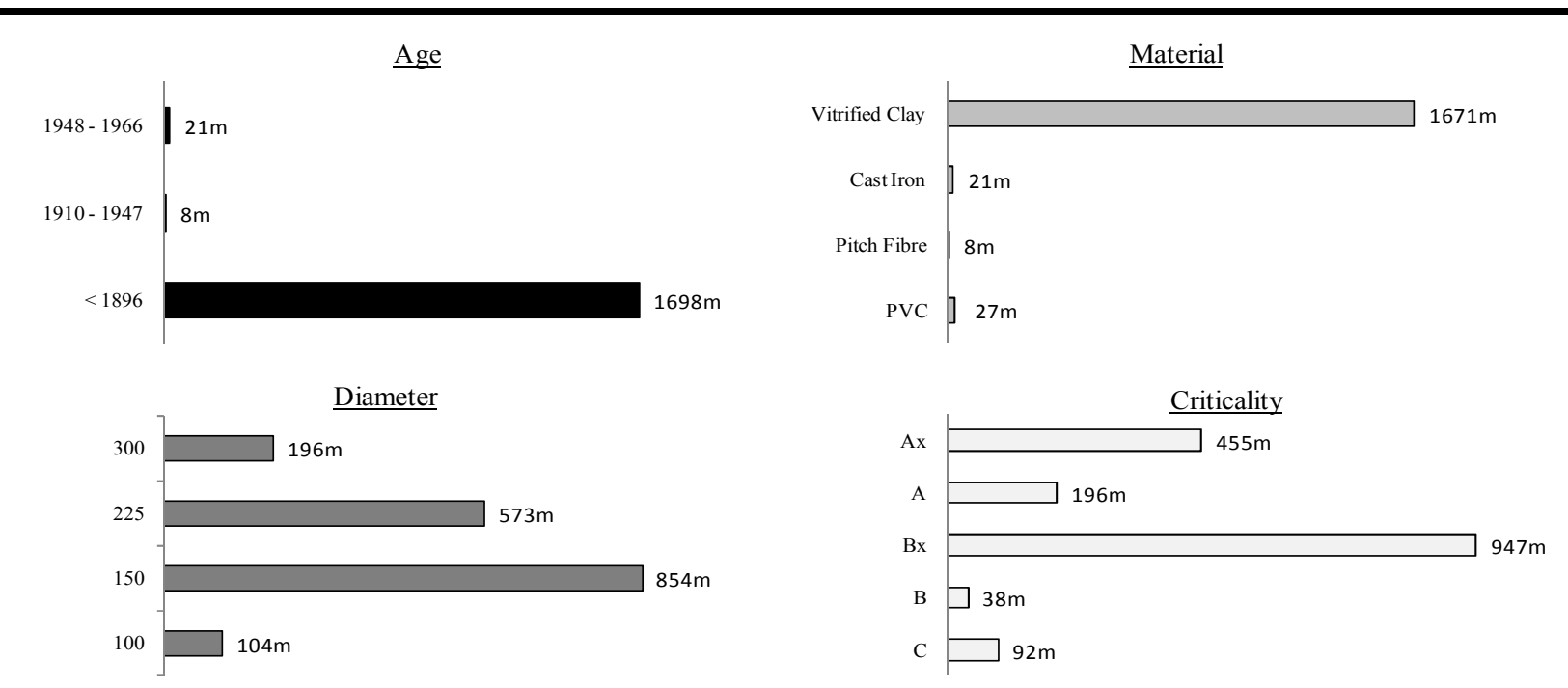

Figure 3: Sewer characteristics

Upon establishing the raw data within the optimisation environment, a Macro driven process is performed to incorporate the three objective functions within the model environment. This process permits the live evaluation of different rehabilitation solutions as the GA iteratively searches the solution spaces for optimal rehabilitation strategies. For any given rehabilitation solution, the following aspects, which constitute the main objective functions, are evaluated; pre and post intervention structural condition scores $\left(\mathrm{S}^{0}\right.$ and $\left.\mathrm{S}^{1}\right)$, raw rehabilitation cost and mobilisation cost $\left(\mathrm{C}_{\text {raw }}\right.$ and $\left.\mathrm{C}_{\text {mob }}\right)$, post rehabilitation peak structural condition score $\left(\mathrm{S}_{\text {peak }}\right)$ and the asset criticality weighting $\left(\mathrm{C}_{\text {weight }}\right)$. A 
To be cited as: Ward, B. and D.A. Savić (2013) A multi-objective optimisation model for sewer rehabilitation considering critical risk of failure, Water Science \& Technology, Vol 66, No 11, pp 2410-2417.

tabulated example of an evaluated output from a rehabilitation strategy for 10 assets is produced in Table 2.

Table 2: Sewer rehabilitation strategy analysis

\begin{tabular}{|c|c|c|c|c|c|c|c|c|c|c|c|}
\hline \multicolumn{3}{|c|}{ Asset Data } & \multicolumn{3}{|c|}{ Structural Condition } & \multicolumn{3}{|c|}{ Construction Cost } & \multicolumn{3}{|c|}{ Critical Risk } \\
\hline ID & Diameter & $\begin{array}{c}\text { Criticality } \\
\text { Code }\end{array}$ & $\mathrm{S}^{0}$ & $\mathrm{~S}^{1}$ & $\Delta \mathrm{S}$ & Craw & Cmob & $\mathrm{C}$ & Speak & Cweight & $\mathrm{R}$ \\
\hline 1 & 100 & $\mathrm{~A}$ & 80 & 80 & 0 & $£ 0$ & $£ 0$ & $£ 0$ & 80 & 4 & 320 \\
\hline 2 & 225 & $\mathrm{C}$ & 770 & 0 & 770 & $£ 6,863$ & $£ 500$ & $£ 7,363$ & 0 & 1 & 0 \\
\hline 3 & 225 & $\mathrm{Bx}$ & 3845 & 0 & 3845 & $£ 35,497$ & $£ 500$ & $£ 35,997$ & 0 & 3 & 0 \\
\hline 4 & 225 & A & 165 & 165 & 0 & $£ 0$ & $£ 0$ & $£ 0$ & 165 & 4 & 660 \\
\hline 5 & 150 & B & 314 & 0 & 314 & $£ 3,600$ & $£ 500$ & $£ 4,100$ & 0 & 2 & 0 \\
\hline 6 & 300 & $A x$ & 290 & 0 & 290 & $£ 3,930$ & $£ 500$ & $£ 4,430$ & 0 & 5 & 0 \\
\hline 7 & 225 & $\mathrm{Ax}$ & 220 & 40 & 180 & $£ 1,183$ & $£ 500$ & $£ 1,683$ & 30 & 5 & 150 \\
\hline 8 & 300 & A & 80 & 80 & 0 & $£ 0$ & $£ 0$ & $£ 0$ & 80 & 4 & 320 \\
\hline 9 & 150 & $\mathrm{C}$ & 241 & 0 & 241 & $£ 3,668$ & $£ 500$ & $£ 4,168$ & 0 & 1 & 0 \\
\hline 10 & 150 & $\mathrm{~A}$ & 1137 & 0 & 1137 & $£ 16,694$ & $£ 500$ & $£ 17,194$ & 0 & 4 & 0 \\
\hline \multicolumn{3}{|c|}{ Objective function outputs } & & & 6,777 & & & $£ 74,934$ & & & 1,450 \\
\hline
\end{tabular}

In this example the rehabilitation strategy scores the following for each of the objective functions;
1. Structural condition improvement
$(\Delta \mathrm{S}) \quad=6,777$
2. Rehabilitation construction cost
(C) $\quad=£ 74,934$
3. Critical risk of failure
(R) $\quad=1,450$

The values of the objective function items 1 and 3, condition improvement and critical risk of failure, are meaningless numbers except where a Pareto optimal trade-off curve is presented to the user. Halhal et al. (1997) explained that each point on the Pareto optimal curve is not dominated by any other point, i.e. in going from one point to another it is not possible to improve on one criterion without making at least one of the other criteria worse. The GANetXL optimisation model used in this analysis (Savić et al., 2011) evaluates each of the objective functions separately. In contrast, a single-objective optimisation problem is solved by finding a single optimal solution. Therefore, when a multi-objective problem is solved successfully, wide arrays of solutions are presented as Pareto optimal trade-off curves. In this instance, these solutions show the trade-off between the three objective functions being considered. Thereby, a range of Pareto optimal solutions is presented to the user to aid in their selection of the most suitable rehabilitation strategy. It is most common for decision makers to evaluate solutions in terms of cost. Therefore, the two non monetary based objective functions that are evaluated in this case study, are presented graphically along the y-axis against a common financial base-line along the $\mathrm{x}$-axis of Figure 4. Figure 4 provides a direct comparison between conventional engineering solutions that have been produced for the catchment and those solutions identified by the optimisation tool. 




(1) Structural Score Improvement

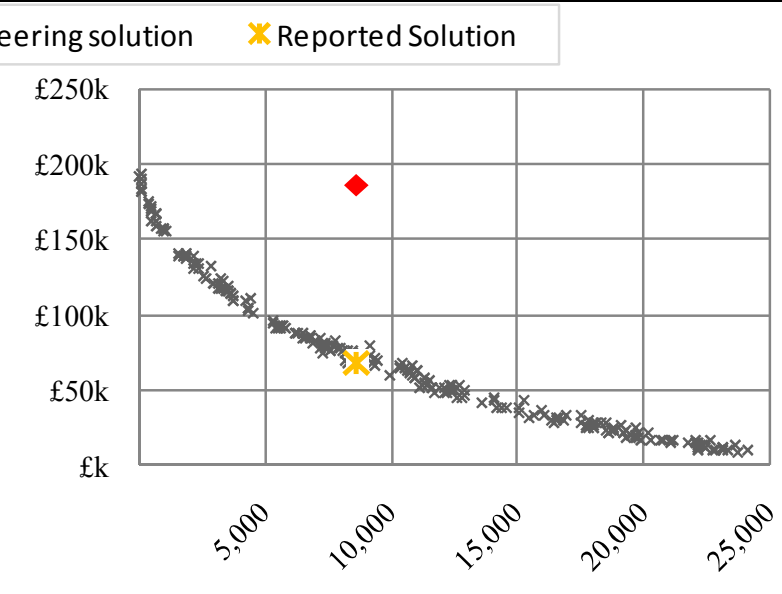

(3) Critical Risk of Failure

Figure 4: Engineering and optimisation solution comparison

It can be seen that a conventional approach to sewer rehabilitation produced a solution to the problem at an estimated construction value of $£ 186,541$. In terms of Objective Function (1) Structural condition improvement, this solution was evaluated at 16,650. However, an equivalent solution in-terms of structural condition improvement, is identified by the optimisation model at a cost of only $£ 82,319$. Hereby, acknowledging a potential saving in excess of $£ 100,000$ between the conventional engineering solution and that of the optimisation model. Similarly, an even greater saving of $£ 116,000$ can be achieved for the delivery of an equally beneficial solution in-terms of Objective Function (3) Critical risk of failure, when it is compared using a financial base-line of Objective Function (2) Construction cost.

Figure 4 illustrates that the GA optimisation tool is capable of producing a wide array of nondominated solutions for the user to select; ranging from an investment value of $£ 200,000$ to $£ 0$, which represent the options for fixing all or none of the observed structural defects respectively. Alternatively, the user can use these trade-off solution curves to fix an acceptable level of critical risk within each catchment by delivering the combination of rehabilitation solutions that reduce the critical risk of failure to below this pre-defined level. This advance level of solution expenditure vision and improved understand of rehabilitation solution benefit, provides both planners and engineers alike with a unique platform for engineering creativity and advanced financial planning capabilities.

\section{CONCLUSIONS}

Sewerage systems are an essential element of the urban water infrastructure system. The rehabilitation and maintenance work associated with these assets form a large part of a utility company's annual expenditure. Therefore, as these infrastructure systems age, the pressure to effectively manage and rehabilitate these systems is also increasing. The result is a demand in the need for engineers to develop rehabilitation strategies that meet multiple conflicting objectives; maximisation of the overall structural condition improvement of the network (Objective Function 1), minimisation of construction costs (Objective Function 2), and minimisation of the risk of critical asset failure (Objective Function 3). Conventionally, it has only been possible to develop sewer rehabilitation strategies on an asset-byasset evaluation basis which calls on engineering best practise guidance to determine when a sewers' condition is deemed worthy of rehabilitative action. In addition, the lack in human ability to evaluate problems of a conflicting and complex nature makes the optimal specification of rehabilitation strategies within a catchment an almost impossible manual task to achieve. However, via the 
To be cited as: Ward, B. and D.A. Savić (2013) A multi-objective optimisation model for sewer rehabilitation considering critical risk of failure, Water Science \& Technology, Vol 66, No 11, pp 2410-2417.

introduction of a multi-objective optimisation tool to the problem, a unique methodology capable of quantifiably appraising optimal rehabilitation strategies is developed.

An appraisal of the models effectiveness and suitability has been conducted on a catchment case study provided by South West Water, UK. The catchment rehabilitation strategy, produced manually using engineering best practice, was evaluated by the rehabilitation cost model developed in this study at $£ 186,500$. In comparison, the optimisation model identified equally beneficial solutions, as defined by the aforementioned Objective Functions 1 and 3, at a cost of only $£ 82,300$ and $£ 69,000$ respectively. Whilst the global optimality of the solutions identified cannot be guaranteed, the model clearly demonstrates the ability to converge towards optimal solutions which would otherwise be over-looked through manual interpretation of the data alone. Therefore, given the resounding improvement over the manual specification of sewer rehabilitation strategies, coupled with the ability of the optimisation tool to use widely available condition data, this study further reinforces the need to effectively integrate such Hydroinformatic tools into business as usual within the UK sewerage industry.

\section{Acknowledgements}

The authors gratefully acknowledge financial support from EPSRC through their funding of the STREAM Industrial Doctorate Centre, and from the project sponsor AECOM.

\section{References}

Adey, B., Bernard, O., \& Gerard, B. (2003). Risk based replacement strategies for redundant deteriorating reinforced conrete pipe networks. Proceedings of 17th International Conference on Strucutural Mechanics in Reactor Technology SMiRT. Prague.

Beradi, L., Gioustolisi, O., Savić, D. A., \& Kapelan, Z. (2008). An effective multi-objective approach to prioritisation of sewer pipe inspection. 11th International Conference on Urban Drainage .

Bicik, J., Morley, M., \& Savić, D. (2008). A Rapid Optimization Prototyping Tool For Spreadsheet-based Models. Proceedings of the 10th Annual Water Distribution Systems Analysis Conference, (pp. 472-482). Kruger National Park, South Africa.

Bicik, J., Morley, M., \& Savić, D. (2006). GANetXL User Manual. Retrieved 02 10, 2010, from Univeristy of Exeter, Centre for Water Systems: http://centres.exeter.ac.uk/cws/technology/ganetxl-addin

Black, M., Brint, A. T., \& Brailsford, J. R. (2005). A semi-Markov approach for modelling asset deterioration. Journal of the Operational Research Society, 56 (11), 1241 - 1249.

Cobra Technologies. (2009, 12 31). CIMS V9 User Manual. Retrieved 08 17, 2010, from Cobratec: http://www.cobratec.com/brochures/CIMS_V9_manual.pdf

Davies, J. P., Clarke, B. A., Whiter, J. T., \& Cunningham, R. J. (2001) Factors influencing the structural deterioration and collapse of rigid sewer pipes. Urban Water, 3, 73-89.

Elachachi, S. M., \& Breysse, D. (2007). Sewer assets maintenance management by three approaches: relative risk based analysis, Markov process and probabilistic neural networks. International Forum on Engineering Decision Making. Shoal Bay, Australia.

Feeney, C. S., Thayer, S., Bonomo, M., \& Martel, K. (2009) Condition Assessment of Wastewater Collection Systems White Paper on Condition Assessment of Wastewater Collection Systems.

Fenner, R. A., Sweeting, L., \& Marriott, M. J. (2000). A new approach for directing proactive sewer maintenance. Proceedings of the Institution of Civil Engineers. Water, maritime and energy, (pp. 67-77). London.

Halhal, D., Walters, G., Ouazar, D., \& Savić, D. (1997). Water Network Rehabilitation with a Structured Messy Genetic Algorithm. Water Resources Planning and Management, 123 (3), 137-146.

Innovyze (2010). InfoNet. Retrieved 07 20, 2010, from MWH Soft: http://www.mwhsoft.com/products/infonet/

Intergraph. (2007). G/Water Product Sheet. Retrieved 08 17, 2010, from Intergraph: http://www.intergraph.com/literature/gwater.pdf

Kaplan, S., \& Garrick, B. J. (1981). On The Quantitative Definition of Risk. Risk Analysis , 1 (1), 11-26.

Kathula, V. S., Pumphrey, N. D., Roberts, F. L., \& Nassar, R. (1999). Integrating Sewer Condition with Sewer Management. Proceedings of No Dig 1999 Conference (pp. 91 -102). Orlando: North American Society for Trenchless Technology.

Kim, J. H., \& Mays, L. W. (1994). Optimal Rehabilitation Model for Water-Distribution Systems. Water Resources Planning and Management, $120(5), 674-692$.

Malandain, J., Le Gauffre, P., \& Miramond, M. (1998). Organising a decision support system for infrastructure maintenance: Application to water supply systems. 1st International conference on new information technologies for decision making in civil engineering. Montreal, Canada.

Murphy, L. J., Simpson, A. R., \& Dandy, G. C. (1993). Pipe network optimization using an improved genetic algorithms. University of Adelaide: Research Report No. R 109, Department of Civil Engineering.

Newton, L. A. \& Vanier, D. J. (2006) MIIP Report : The State of Canadian Sewers - Analysis of Asset Inventory and Condition. 
To be cited as: Ward, B. and D.A. Savić (2013) A multi-objective optimisation model for sewer rehabilitation considering critical risk of failure, Water Science \& Technology, Vol 66, No 11, pp 2410-2417.

Nicklow, J. W., Reed, P., Savić, D. A., Dessalegne, T., Harrell, L., Chan-Hilton, A., et al. (2010). State of the Art for Genetic Algorithms and Beyond in Water Resources Planning and Management. ASCE Journal of Water Resources Planning and Man-agement , 136 (4), $412-432$.

Ofwat. (2009). Financial performance and expenditure of the water companies in England and Wales 2008-09. Retrieved 06 08, 2010, from Ofwat: http://www.ofwat.gov.uk/regulating/rpt fpr_2008-09.pdf

Read, G. F., \& Vickridge, I. G. (1997). Sewers - Rehabilitation and New Construction - Repair and Renovation. Elsevier.

Savić, D. A., Bicik, J., \& Morley, M. S. (2011). GANETXL. A DSS Generator for Multiobjective Optimisation of Spreadsheet-Based Models. 26, pp. 551-561. Environmental Modelling Software.

Shamir, U., \& Howard, C. (1979). An Analytical Approach to Scheduling Pipe Replacement. AWWA , 71 (5), 248-258.

Ugarelli, R. and Federico, V. D. (2010) Optimal Scheduling of Replacement and Rehabilitation in Wastewater Pipeline Networks. Water

Resources, (June), 348-356.

Ward, B., \& Savić, D. A. (2011). Multi-objective Optimisation for Sewer Rehabilitation Investment Planning. 34th International Association for Hydro-Environment Engineering and Research World Congress (accepted for publication). Brisbane.

WinCan. (2008). WinCan Map2008. Retrieved 08 17, 2010, from WinCan: http://www.cdlab.ch/wincan/administration.php?id=128

Woodburn, J., Lansey, K. E., \& Mays, L. W. (1987). Model for the optimal rehabilitation and replacement of water distribution system components. National Conference on Hydraulic Engineering (pp. 606 - 611). New York: ASCE.

WRc. (2004). Sewerage Risk Management - Manual, Fourth Edition. UK: Water Research Centre. 